\title{
Safety and effectiveness of a glistening-free single-piece hydrophobic acrylic intraocular lens (enVista)
}

\author{
Mark Packer' \\ Luther Fry ${ }^{2}$ \\ Kevin T Lavery ${ }^{3}$ \\ Robert Lehmann ${ }^{4}$ \\ James McDonald ${ }^{5}$ \\ Louis Nichamin ${ }^{6}$ \\ Brian Bearie ${ }^{7, \dagger}$ \\ Jon Hayashida ${ }^{8}$ \\ Griffith E Altmann ${ }^{8}$ \\ Omid Khodai ${ }^{8}$ \\ 'Department of Ophthalmology, \\ Oregon Health and Science University, \\ Eugene, OR, USA; ${ }^{2}$ University of \\ Kansas Medical Center, Kansas City, \\ KS, USA; ${ }^{3}$ Wayne State University, \\ Detroit, MI, USA; ${ }^{4}$ Baylor College \\ of Medicine, Houston, TX, USA; \\ ${ }^{5}$ University of Arkansas for Medical \\ Sciences, Little Rock, AR, USA; ' ${ }^{6}$ Laurel \\ Eye Clinic, Brookville, PA, USA; \\ ${ }^{7}$ Grand Rapids Eye Institute, Grand \\ Rapids, MI, USA; ${ }^{8}$ Bausch \& Lomb, \\ Aliso Viejo, CA, USA
}

†Brian Bearie passed away on March 9, 201 I

Correspondence: Mark Packer Mark Packer MD Consulting, Inc. 777 S. Flagler Drive, Suite I700-W West Palm Beach, FL 3340I, USA

$\mathrm{Tel}+\mathrm{I} 5419150291$

$\mathrm{Fax}+\mathrm{I} 8888409392$

Email mark@markpackerconsulting.com
This article was published in the following Dove Press journal:

Clinical Ophthalmology

25 September 2013

Number of times this article has been viewed

Purpose: To evaluate the safety and effectiveness of a single-piece hydrophobic acrylic intraocular lens (IOL; enVista model MX60; Bausch \& Lomb, Rochester, NY, USA) when used to correct aphakia following cataract extraction in adults.

Methods: This was a prospective case series (NCT01230060) conducted in private practices in the US. Eligible subjects were adult patients with age-related cataract amenable to treatment with standard phacoemulsification/extracapsular cataract extraction. With follow-up of 6 months, primary safety and effectiveness end points included the rates of US Food and Drug Administration (FDA)-defined cumulative and persistent adverse events and the percentage of subjects who achieved best-corrected visual acuity (BCVA) of 20/40 or better at final visit. To evaluate rotational stability, subjects were randomized (1:1:1:1) to have the lens implanted in one of four axis positions in $45^{\circ}$ increments.

Results: A total of 122 subjects were enrolled. The rate of cumulative and persistent adverse events did not significantly exceed historical controls, as per FDA draft guidance. At the final postoperative visit, all subjects (100\%) achieved a BCVA of 20/40 compared with the FDA historical control of $96.7 \%$. Rotation of the IOL between the two final follow-up visits was $\leq 5^{\circ}$ for $100 \%$ of eyes, and refractive stability was demonstrated. A low evaluation of posterior capsule opacification score was demonstrated, and no glistenings of any grade were reported for any subject at any visit.

Conclusion: This study demonstrated the safety and effectiveness of the MX60 IOL. Favorable clinical outcomes included preserved BCVA, excellent rotational and refractive stability, no glistenings, and a low evaluation of posterior capsule opacification score.

Keywords: cataract surgery, enVista, glistenings, intraocular lens, hydrophobic acrylic

\section{Introduction}

Modern cataract surgery is characterized by a growing array of options to customize the procedure and optimize outcomes. During the past decade, both intraocular lens (IOL) design and materials have advanced substantially, ${ }^{1}$ resulting in improved visual function and fewer complications. One development is that of a novel, hydrophobic acrylic polymer, which has been tested and approved by the US Food and Drug Administration (FDA) in the form of a three-piece lens (XACT X-60/X-70; Advanced Vision Science, Goleta, CA, USA). More recently, the MX60 IOL (enVista model MX60; Bausch \& Lomb, Rochester, NY, USA) was introduced, in which both the optic and haptics are formed from the same hydrophobic acrylic polymer. In 2012, the MX60, a glisteningfree lens, was approved by the FDA as a single-piece hydrophobic acrylic IOL. This paper presents the FDA clinical trial results investigating this lens. 


\section{Materials and methods}

\section{Study design}

This was a prospective case series evaluating primary safety and efficacy of a new IOL conducted at six sites in the US (NCT01230060). Planned enrollment was up to 125 eyes of 125 subjects. The study protocol was reviewed and approved by a governing institutional review board prior to initiation of the study at each center. The board operated in accordance with the principles and requirements described in US 21 Code of Federal Regulations, Part 56.

The primary safety end point of this study was the occurrence of adverse events (AEs), described in terms of statistical incidence rates. The primary effectiveness end point was the percentage of subjects achieving bestcorrected distance visual acuity (BCVA) of 20/40 or better at visit four (120-180 days postoperatively). Although the study involved no formal secondary objectives for claims of safety or effectiveness, other effectiveness outcomes studied included rotational stability and manifest refraction stability. In addition, other variables were explored to understand fully the success of the intervention.

\section{Eligibility criteria}

Adult subjects with clinically documented diagnoses of age-related cataracts amenable to treatment with standard phacoemulsification/extracapsular cataract extraction with otherwise clear ocular media were enrolled in this study. Additionally, subjects needed to have met the inclusion and exclusion criteria listed in Table 1. Only one eye of each patient was eligible for inclusion in this study.

\section{Lens material and design features}

The MX60 lens optic and haptics are composed of a hydroxyethyl methacrylate-polyethylene glycol phenyl ether acrylate-styrene copolymer, cross-linked with ethylene glycol dimethacrylate. This material has an ultravioletabsorbing chromophore. Surface-energy and contact-angle measurements have demonstrated that the IOL material is a true hydrophobic polymer, with water contact-angle values comparable with those of other hydrophobic acrylic IOLs. ${ }^{2}$

The surface hardness of the material is $11.0 \mathrm{MPa}$, which compares favorably with the $0.24,0.68$, and 0.43 found for AcrySof(Alcon Laboratories, Fort Worth, TX, USA), Acryfold (Hoya, Santa Clara, CA, USA), and Sensar (Abbott Medical Optics, Abbott Park, IL, USA) lens materials, respectively. ${ }^{3}$ The relatively greater surface hardness and rigidity of the material results in a less compliant lens that is relatively impervious to scratching and deformation. The reduced compliance also
Table I Eligibility criteria

Inclusion criteria

- Clinically documented diagnosis of age-related cataract that was considered amenable to treatment with standard phacoemulsification/ extracapsular cataract extraction

- Clear intraocular media other than cataract

- Age $\geq 18$ years

- Able to read, understand, and provide written informed consent

- Required a lens power from 16.5 to $24.0 \mathrm{D}$

- Had a BCVA equal to or worse than 20/40 in the study eye, with or without a glare source

- Had a BCVA projected to be better than 20/30 after IOL implantation in the study eye

Exclusion criteria

- Any anterior segment pathology for which extracapsular phacoemulsification cataract surgery may be contraindicated

- Uncontrolled glaucoma or current treatment for glaucoma in either eye

- Previous retinal detachment or clinically significant retinal pathology in the operative eye

- Proliferative or clinically significant nonproliferative diabetic retinopathy in either eye

- Congenital bilateral cataracts

- Marked microphthalmos or aniridia in either eye

- BCVA of 20/200 (logMAR I.0,6/60) or worse in the fellow eye

- Previous ocular surgery in the operative eye

- Previous recipient of a study IOL in the fellow eye

- Inability to achieve a minimum pharmacologic pupil dilation of $5.0 \mathrm{~mm}$

- Preoperative corneal astigmatism of a magnitude that required surgical treatment at any time during the study

- Preoperative corneal astigmatism greater than I.5 D

Abbreviations: BCVA, best-corrected visual acuity; IOL, intraocular lens; $\log M A R$, logarithm of minimum angle of resolution.

results in a requirement for greater force in folding the lens for insertion into the injection cartridge. Of note, the rigidity of the material is highly temperature-sensitive, so a slightly warmer lens will fold more easily. The low compliance of the material carries implications for the design of suitable injection devices for this lens. The design and surface characteristics of the cartridge and plunger must take into account the characteristics of the lens material. The enVista MX60 IOL has been validated with the INJ100 injector (Bausch \& Lomb), which has the same cartridge-tip dimensions as the Monarch D cartridge (Alcon Laboratories). As with all IOLs, the material characteristics and design of the injector result in a range of appropriate incision sizes. The optimal incision size will also depend on the surgical technique (eg, a smaller incision with a wound-assist technique).

The biconvex lens optic has a body diameter of $6.0 \mathrm{~mm}$ and an overall length (diameter) of $12.5 \mathrm{~mm}$ (Figure 1A). The MX60 IOL has aberration-free aspheric optics and modified C-loop haptics. The optic and haptics are lathed and milled from a single button made from the proprietary soft 

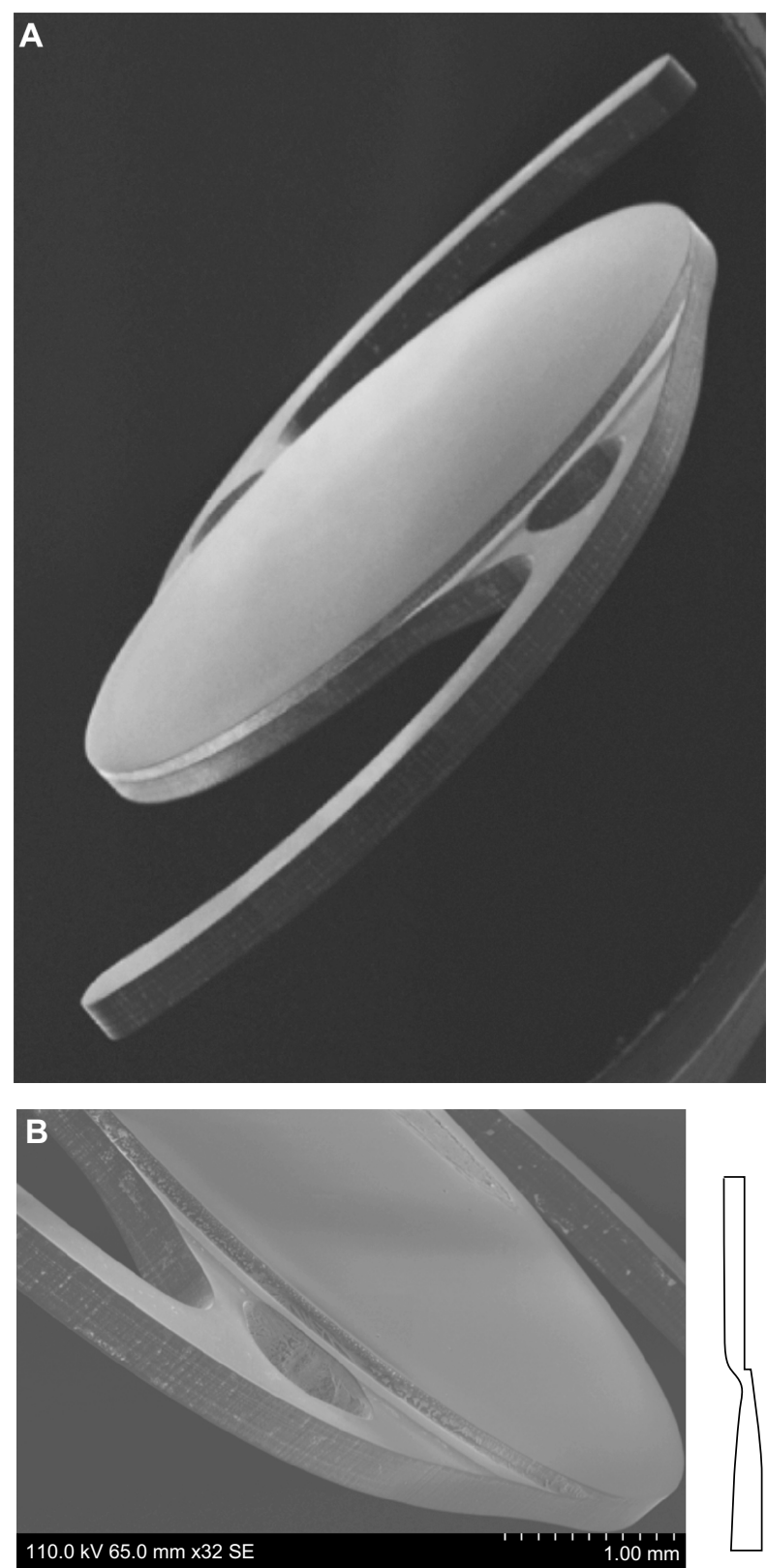

Figure I (A) Design of the MX60 intraocular lens (Bausch \& Lomb, Rochester, NY, USA). (B) Posterior surface facing toward the right side of the page. The haptics are offset anteriorly with respect to the optic body, which enables consistent posterior movement of lens optic under haptic compression. Images courtesy of David Spalton, FRCS, FRCP, FRCOphth.

hydrophobic acrylic polymer. Lens power is uniform from center to edge, with enhanced contrast sensitivity. ${ }^{4-6}$ It has a sharp $360^{\circ}$ square posterior edge, which is intended to minimize posterior capsule opacification (PCO) (Figure 1B). ${ }^{7}$ The haptics contain fenestrations to prevent transfer of stresses from the haptic to the optic.

\section{Study procedures}

Eligible subjects who provided written informed consent underwent a preoperative visit (up to 60 days before surgery), the operative visit (day 0), and four postoperative visits (visit one, 1-2 days postoperatively; visit two, 7-14 days; visit three, 30-60 days; and visit four, 120-180 days). The preoperative visit included determinations of keratometry, axial length, pupil diameter, target postoperative refraction, and IOL power. On the day of surgery, eligibility was reconfirmed and subjects were randomized $(1: 1: 1: 1)$ to have the lens implanted in one of four axis positions $\left(45^{\circ}, 90^{\circ}\right.$, $135^{\circ}$, or $180^{\circ}$ ) to evaluate subsequent rotational stability. Study lenses were available in powers ranging from $16.5 \mathrm{D}$ to $24.0 \mathrm{D}$ in $0.5 \mathrm{D}$ increments. The estimated A-constant for the IOL Master (Carl Zeiss Meditec, Dublin, CA, USA) was 119.1 , with an anterior chamber depth of $5.38 \mathrm{~mm}$.

Postoperative assessments included evaluation of BCVA, manifest refraction, rotational stability of the lens, optic tilt and decentration, IOL glistenings grade, evaluation of $\mathrm{PCO}$ (EPCO) score, and monitoring for AEs. Details on selected measurements are described as follows.

\section{Adverse events}

AEs were recorded, rated as to severity and relationship to the device, and compared with FDA historical control values, as per an FDA draft guidance document ${ }^{8}$ (FDA historical controls).

\section{Rotational stability}

Rotational stability was assessed by an independent third party using the method described by Wolffsohn and Buckhurst, ${ }^{9}, 10$ with digitally captured images. A line is drawn connecting the IOL orientation marks to document the alignment of these markings. To normalize for any rotation of the eye at the slit lamp between visits, the axis of a line joining two consistent conjunctival vessels or iris features on opposite sides of the pupil margin is compared with the line drawn.

\section{Intraocular lens tilt and decentration}

IOL tilt was determined using Purkinje images by the method described by Guyton et al. ${ }^{11}$ The technique used to evaluate IOL decentration was described by Wolffsohn and Buckhurst, ${ }^{9}$ using digitally captured images.

\section{Posterior capsule opacification evaluation}

PCO was quantified using the EPCO 2000 software (software is not commercially available but is available free for download at http://www.epco2000.de/). ${ }^{12}$ The EPCO system has been found to be a reliable and repeatable method of determining the opacity density and extent of PCO. ${ }^{12}$ This system is a morphologic assessment of PCO in which the 
geographical extent and density of backscatter on retroilluminated images determines the overall EPCO score. The density of opacification is graded on a scale of 0 to 4 ( $0=$ none, $1=$ minimal, $2=$ mild, $3=$ moderate, $4=$ severe $)$. The individual EPCO score is calculated by multiplying the opacification grade by the fraction of capsule area involved behind the IOL optic.

\section{Evaluation of glistenings}

Glistenings, which are fluid-filled microvacuoles that form within the IOL optic when in an aqueous environment, ${ }^{13}$ were evaluated via retroillumination slit-lamp examination using a photographic grading scale. The grading scale was, in order of severity, none, trace, mild, moderate, severe, and very severe.

\section{Statistical methods and analyses}

The analysis cohorts included the safety, best-case, and consistent sets. All subjects comprised the safety set. The best-case set, as defined by the International Organization for Standardization 11979-1, ${ }^{14}$ consisted of those subjects with no major protocol violations, preoperative ocular pathology, macular degeneration at any time, or previous refractive surgery. The main effectiveness outcomes were derived from the best-case set. The consistent set was comprised of subjects with no major protocol violations who had data at every follow-up visit.

As the primary safety end point, AEs were described as incidence rates. As the primary effectiveness variable, Snellen values for BCVA were summarized categorically at each visit. The proportion of eyes with a BCVA of 20/40 or better at visit four (with 95\% confidence intervals) was compared with FDA historical controls ${ }^{8}$ using an exact binomial test. Manifest refraction, lens rotation, decentration, tilt, PCO, and glistenings were captured as categorical variables and tabulated at specific visits. Where statistical tests for significance were performed, a significance level of 0.05 was adopted. All tests were two-sided unless otherwise specified.

\section{Results}

\section{Demographics}

Demographic data are summarized in Table 2 . The majority of patients $(85.2 \%)$ in this study were between the ages of 60 and 79 years. A total of 122 subjects were enrolled and randomized, comprising the safety-analysis set; 119 formed the best-case analysis set for effectiveness evaluations, and 112 formed the consistent analysis set. The basis for the
Table 2 Subject demographics $(n=122)$

\begin{tabular}{ll}
\hline Variable & \\
Sex, $\mathrm{n}(\%)$ & $53(43.4)$ \\
$\quad$ Male & $69(56.6)$ \\
$\quad$ Female & \\
Age in years at surgery date \pm SD (range) & $69.0 \pm 8.0$ (46 to 93) \\
$\quad$ Overall & $69.1 \pm 7.6(49$ to 83$)$ \\
Male & $69.0 \pm 8.3$ (46 to 93) \\
Female & \\
Subjects by age in years, $\mathrm{n}(\%)^{*}$ & $13(10.7)$ \\
$\quad<60$ & $50(4 \mathrm{I} .0)$ \\
$60-69$ & $54(44.3)$ \\
$70-79$ & $5(4.1)$ \\
$\geq 80$ & \\
Ethnicity, $\mathrm{n}(\%)^{*}$ & $\mathrm{I}(0.8)$ \\
African American & $\mathrm{I} 19(97.5)$ \\
Caucasian & $2(\mathrm{I} .6)$ \\
Hispanic & \\
\hline
\end{tabular}

Note: *Percentages may not total 100 due to rounding.

Abbreviation: SD, standard deviation.

distribution of subjects into each analysis cohort is shown in Figure 2.

\section{End points}

\section{Safety}

All 122 subjects enrolled underwent cataract extraction via phacoemulsification and implantation of the MX60 IOL using the Accuject injection system (Medicel, Wolfhalden, Switzerland). For all subjects, the primary incision type was clear cornea, and no sutures were required. Intraoperatively, no additional surgical procedures were required. One subject $(0.8 \%)$ experienced mild miosis and loss of subincisional iris pigment during surgery.

The FDA-defined AEs were categorized as either cumulative (occurred at any time through visit four) or persistent (present at visit four). Two subjects (1.6\%) had cumulative AEs of cystoid macular edema. At visit four, one of these subjects $(0.8 \%)$ achieved a BCVA of 20/32. The second subject, who was also the only patient $(0.8 \%)$ observed as having a persistent $\mathrm{AE}$ of cystoid macular edema, achieved a BCVA of 20/20 at visit four. The study met the primary safety end point, as the rate of FDA-defined cumulative and persistent AEs through visit four were statistically at or below FDA historical controls $(P=0.884$ and $P=0.455$, respectively). ${ }^{8}$ Other ocular AEs were of the type and frequency generally observed in patients who have had cataract surgery (Table 3 ). The most frequently reported AEs $(\geq 2 \%$ ) included punctate keratitis (3.3\%), anterior capsule contraction $(2.5 \%)$, conjunctival hyperemia 


\section{Analysis sets}

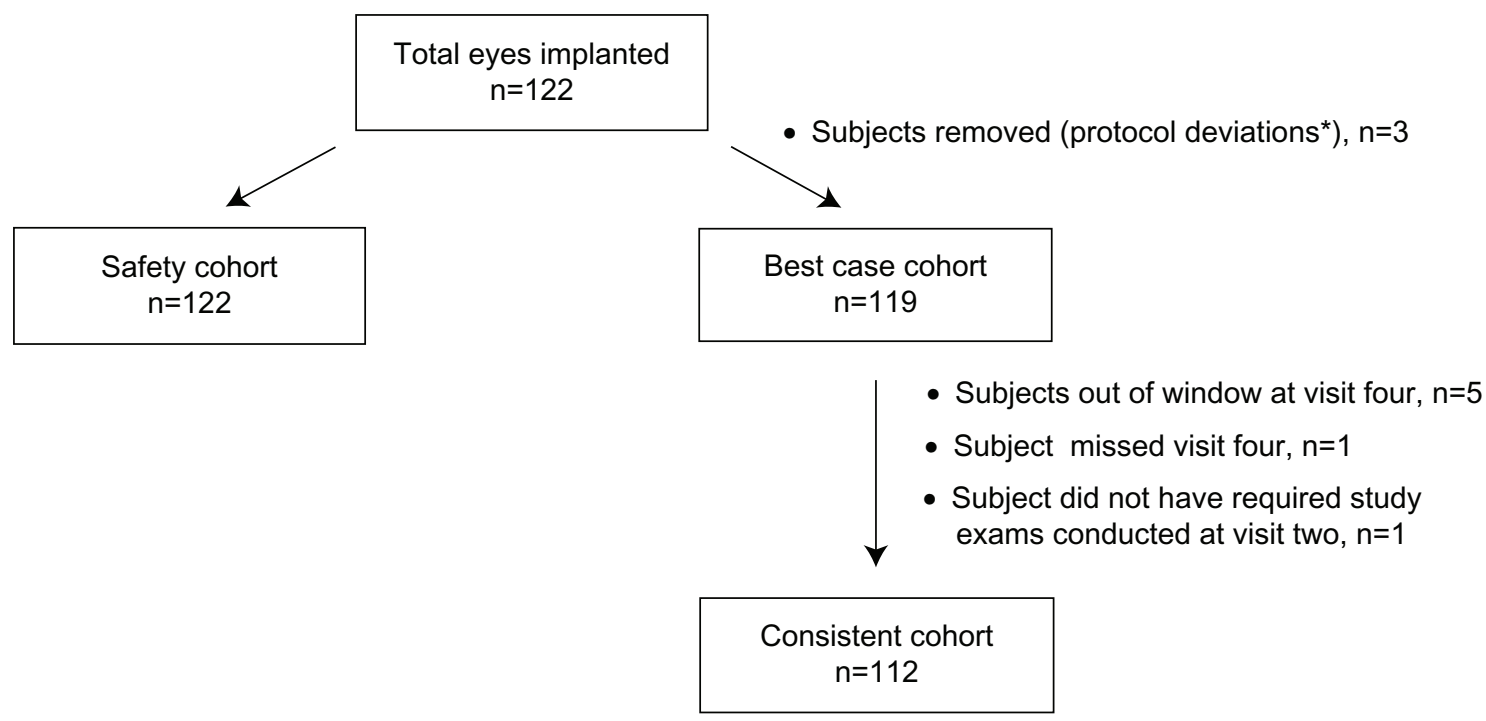

Figure 2 Flowchart showing the basis for distribution of subjects into each analysis cohort.

Note: *Three subjects removed due to preoperative corneal astigmatism > I.5 D (exclusion criterion no I3).

Table 3 Ocular adverse events in safety-analysis set $(n=122)^{*}$

Finding

n (\%)

Anterior capsule contraction

Arcus lipoides

Blepharitis

Conjunctival hemorrhage

Conjunctival hyperemia

Conjunctival edema

Conjunctivitis

Conjunctivitis, allergic

Conjunctivitis, viral

Corneal abrasion

Corneal degeneration

Corneal dystrophy

Diabetic retinopathy

Dry eye

Eye hemorrhage

Eye irritation

Eye pain

Hordeolum

Intraocular pressure increase

Iris hypopigmentation

Iritis

Keratitis, herpetic

Macular degeneration

Miosis

Pinguecula

Punctate keratitis

Vision blurred

Vitreous detachment

Wound secretion

Note: *Includes all ocular adverse events, regardless of relationship to surgery or

device, which occurred during the trial.
(2.5\%), corneal abrasion (2.5\%), dry eye $(2.5 \%)$, iritis $(2.5 \%)$, and vitreous detachment (2.5\%). At visit four, no subject showed evidence of corneal stromal edema or iritis. No serious ocular AEs occurred during this study, and no secondary surgical interventions were required, including explantation.

\section{Best-corrected distance visual acuity}

At visit four (120-180 days postoperatively), 100\% (95\% confidence interval $96.9 \%-100 \%$ ) of subjects in the bestcase analysis set achieved a BCVA of 20/40 or better (Figure 3). This exceeds the FDA historical control value of $96.7 \%{ }^{8}$

\section{Other outcomes}

\section{Rotational stability}

In the best-case analysis set, $92 \%$ of eyes exhibited $\leq 5^{\circ}$ of rotation between operative day and the 4- to 6-month postoperative visit. One hundred percent of subjects had $\leq 5^{\circ}$ of IOL rotation between the 1- to 2-month and the 4- to 6-month postoperative visits, which exceeds the American National Standards Institute (ANSI) ${ }^{15}$ standard of $90 \%$ of subjects with $\leq 5^{\circ}$ of rotation at two consecutive visits at least 3 months apart.

\section{Refractive stability}

For the best-case analysis set, the change in mean manifest refractive spherical equivalent (standard deviation [SD]) was 


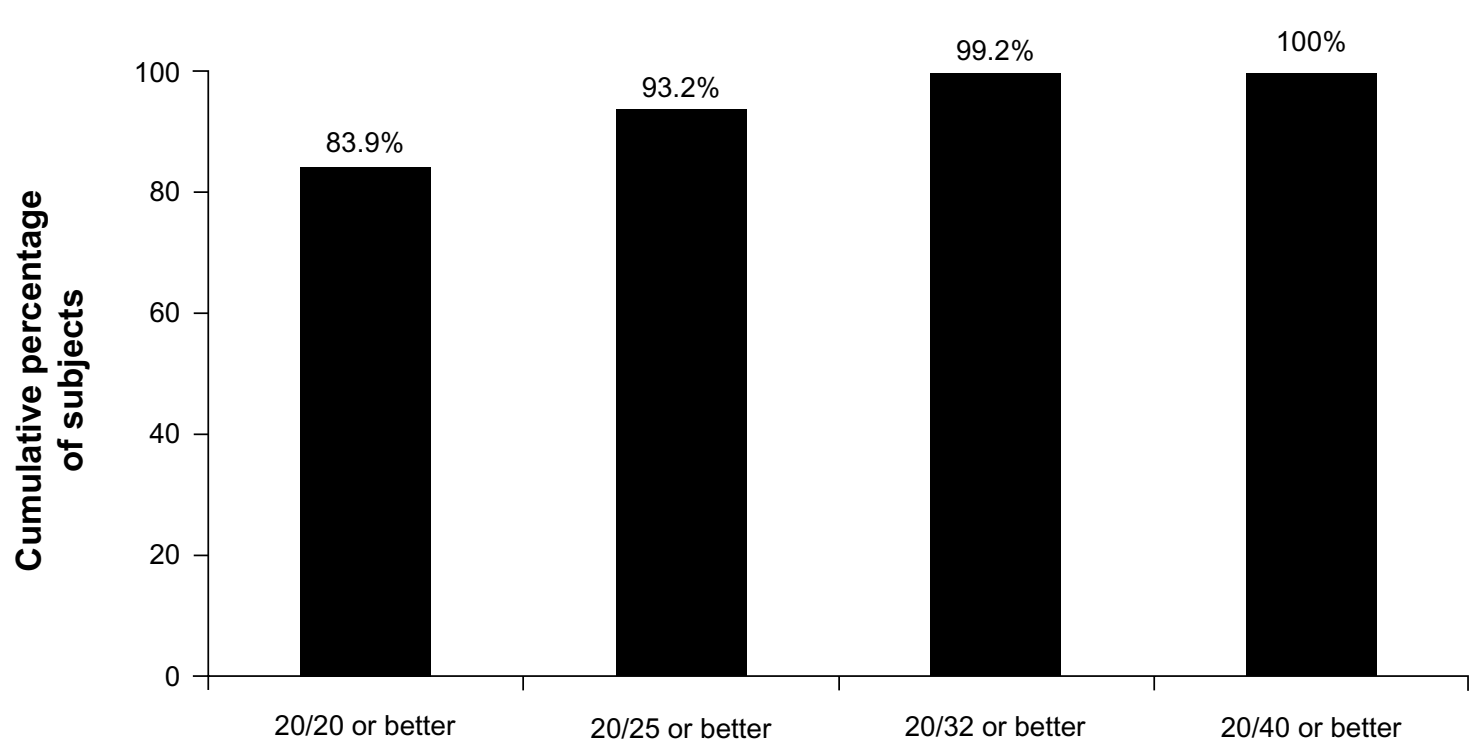

Best-corrected visual acuity

Figure 3 Best-corrected visual acuity (BCVA) at visit four (days I20-180 postoperative); cumulative percentage of subjects $(\mathrm{n}=\mathrm{I} \mid \mathrm{8})$.

reported as $0.01 \mathrm{D}(0.31)$ between visits two and three, and $0.10 \mathrm{D}(0.32)$ between visits three and four, demonstrating good postoperative refractive stability.

\section{Decentration and tilt}

For the best-case analysis set, the IOL exhibited stability, showing similar mean decentration and tilt measurements at each visit. At visit four, the mean (SD) decentration was $0.28 \mathrm{~mm}(0.15)$ and the mean (SD) tilt was $2.67^{\circ}\left(1.73^{\circ}\right)$.

\section{Posterior capsule opacification}

In the safety-analysis set, the mean (SD) EPCO score was $0.032(0.101)$ at visit four.

\section{Glistenings}

All subjects in the safety-analysis set were evaluated for IOL glistenings. No glistenings of any grade were reported for any subject at any visit.

\section{Discussion}

The results of this study demonstrated the safety and effectiveness of the MX60 IOL. The rates of FDA-defined cumulative and persistent AEs through visit four did not statistically significantly exceed FDA historical controls, ${ }^{8}$ meeting the primary safety end point. Two subjects (1.6\%) experienced a cumulative $\mathrm{AE}$ of cystoid macular edema, one of whom $(0.8 \%)$ was also reported as having a persistent $\mathrm{AE}$ of cystoid macular edema. At visit four, all subjects (100\%) achieved a BCVA of $20 / 40$ or better, which exceeded the FDA historical control value of $96.7 \%{ }^{8}$
A number of factors support the stability of the MX60 lens when implanted. The mean tilt and decentration values closely agree with expectations based on results in peerreviewed literature for small-incision cataract surgery with in-the-bag IOL placement and capsulorhexis completely overlying the optic. ${ }^{16}$ Rotational stability for the MX60 IOL was demonstrated, with $100 \%$ of subjects exhibiting $\leq 5^{\circ}$ of rotation, exceeding the ANSI standard for toric IOLs. ${ }^{15}$ In a study investigating the AcrySof IQ Toric IOL (model SA60TT; Alcon Laboratories), approximately $90 \%$ of eyes achieved rotation of $\leq 5^{\circ}$ at $120-180$ days postprocedure. ${ }^{17}$ The MX60 also demonstrated excellent refractive stability over time. This IOL is designed to maximize the haptic contact angle under compression, preventing ovalization and/ or striae of the capsular bag. This achieves better refractive stability, centration, and rotational stability, and ensures that each lens power across the range behaves similarly under compression. These data provide a promising basis on which to develop a toric lens of this material and general design.

The low EPCO score of 0.03 and the excellent outcomes reported during this study may be attributed to the IOL design. The MX60 features step-vaulted haptics that translate the optic posteriorly for direct contact with the capsular bag, in addition to a sharp $360^{\circ}$ square barrier edge to inhibit lens epithelial cell migration. The limited duration of this study may be a factor in long-term assessment of the incidence of PCO.

Although the evaluation of glistenings was not included as an end point in this study, this phenomenon was evaluated. A meta-analysis of published literature on glistenings describes 
them in association with hydrophobic acrylic IOLs, but the hydrophobic acrylic IOLs on the market are not manufactured from the same material and by the same manufacturing process. ${ }^{13}$ In the study reported here, no glistenings of any grade were reported by any subject at any visit. This is consistent with results from a prospective study investigating the XACT (X60; Advanced Vision Science) IOL, a three-piece lens made of the same material as the MX60. In this study, 172 eyes of 142 patients were examined at least once between 1 month and 6 months; 123 eyes of 101 patients were examined at least once between 6 months and 2 years. With this material, no glistenings were detected at any time. ${ }^{18}$

The results of this current study stand in contrast to investigations involving other hydrophobic acrylic IOLs, which report that glistenings appear to increase in severity with time. ${ }^{13,19-21}$ The MX60 lens itself is packaged in physiologic saline to eliminate fluid exchange with the aqueous humor. Prehydration of the MX60 lens to equilibrium water content ensures that it remains glistening-free. As the effect of glistenings on visual performance is unclear, it remains an area of active investigation in ophthalmology. ${ }^{13,21-23}$

In conclusion, the MX60 IOL has demonstrated a good safety profile, excellent BCVA, exceptional refractive and rotational stability, a low incidence of PCO, and no incidence of glistenings when used to correct aphakia following cataract surgery.

\section{Acknowledgments}

The authors would like to acknowledge Dr Phillip Buckhurst, lecturer in optometry, School of Health Professions, College of St Mark and St John, Plymouth, Devon, UK, for his contributions to the manuscript, specifically for providing information on his research on rotational stability and evaluation of PCO scoring. With great sadness, the authors wish to acknowledge the passing of our colleague, Brian Bearie, DO, and recognize his contributions to this article. Funding for writing and editorial support was provided by Bausch \& Lomb, Rochester, NY, USA. Writing and editorial assistance was provided by Paula G Davis, PhD; Ramana Yalamanchili, PhD, MBA; Antoinette Campo of SCI Scientific Communications and Information, Parsippany, NJ, USA; and Stephanie Baba, OD, Alameda, CA, USA.

\section{Disclosure}

Dr Packer is a consultant to Bausch \& Lomb, Abbott Medical Optics, Advanced Vision Science, and Rayner Intraocular Lenses. Dr Bearie was a clinical investigator for Bausch \& Lomb. Dr Fry is a clinical investigator for Bausch \& Lomb.
He has received research grants and honoraria from Bausch \& Lomb. Dr Lavery is a speaker for Alcon and a clinical investigator for Bausch \& Lomb, as well as Tear Science and Glaukos. Dr Lehmann has nothing to disclose. Dr McDonald has received grants from the Advancing Vision Thru Education Foundation. He has served as a consultant in the past, but did not receive any funds for consultancy this year. Dr Nichamin is a speaker, clinical investigator, and consultant for Bausch \& Lomb. He has received honoraria from Bausch \& Lomb. Jon Hayashida, OD, Griffith E Altmann, and Omid Khodai, OD are employees of Bausch \& Lomb.

\section{References}

1. Ashwin PT, Shah S, Wolffsohn JS. Advances in cataract surgery. Clin Exp Optom. 2009;92:333-342.

2. Bausch and Lomb. EnVista HydroStable Acrylic AO Lens [package insert]. Rochester, NY: Bausch and Lomb; 2012.

3. Mentak K, Goldberg E, El-Achchabi A. Nanoindentation studies on hydrophobic acrylic IOLs to evaluate surface mechanical properties. Presented at: 25th Congress of the ESCRS - European Society of Cataract and Refractive Surgeons; September 8-12, 2007; Stockholm, Sweden.

4. Caporossi A, Martone G, Casprini F, Rapisarda L. Prospective randomized study of clinical performance of 3 aspheric and 2 spherical intraocular lenses in 250 eyes. J Refract Surg. 2007;23:639-648.

5. Pepose JS, Qazi MA, Edwards KH, Sanderson JP, Sarver EJ. Comparison of contrast sensitivity, depth of field and ocular wavefront aberrations in eyes with an IOL with zero versus positive spherical aberration. Graefes Arch Clin Exp Ophthalmol. 2009;247: 965-973.

6. Santhiago MR, Netto MV, Barreto J Jr, et al. Wavefront analysis, contrast sensitivity, and depth of focus after cataract surgery with aspherical intraocular lens implantation. Am J Ophthalmol. 2010;149:383-389.

7. Nixon DR, Woodcock MG. Pattern of posterior capsule opacification models 2 years postoperatively with 2 single-piece acrylic intraocular lenses. J Cataract Refract Surg. 2010;36:929-934.

8. Food and Drug Administration. Guidance for industry and for FDA reviewers: intraocular lens guidance document. Available from: http:// www.fda.gov/OHRMS/DOCKETS/98fr/994052gd.pdf. Accessed July 26, 2013.

9. Wolffsohn JS, Buckhurst PJ. Objective analysis of toric intraocular lens rotation and centration. J Cataract Refract Surg. 2010;36:778-782.

10. Buckhurst PJ, Wolffsohn JS, Naroo SA, Davies LN. Rotational and centration stability of an aspheric intraocular lens with a simulated toric design. J Cataract Refract Surg. 2010;36:1523-1528.

11. Guyton DL, Uozato H, Wisnicki HJ. Rapid determination of intraocular lens tilt and decentration through the undilated pupil. Ophthalmology. 1990;97:1259-1264.

12. Tetz MR, Auffarth GU, Sperker M, Blum M, Volcker HE. Photographic image analysis system of posterior capsule opacification. J Cataract Refract Surg. 1997;23:1515-1520.

13. Werner L. Glistenings and surface light scattering in intraocular lenses. J Cataract Refract Surg. 2010;36:1398-1420.

14. International Organization for Standardization. ISO. 11979-11977:2006 Ophthalmic implants - Intraocular lenses - Part 7: Clinical investigations. 2006. Available from: http://www.iso.org/iso/catalogue detail.htm?csnumber=36409. Accessed July 26, 2013.

15. American National Standards Institute. ANSI Z80.30 - 2010. Ophthalmics - toric intraocular lenses. 2010. Available from: http:// webstore.ansi.org/RecordDetail.aspx?sku=ANSI+Z80.30-2010. Accessed July 26, 2013. 
16. de Castro A, Rosales P, Marcos S. Tilt and decentration of intraocular lenses in vivo from Purkinje and Scheimpflug imaging. Validation study. J Cataract Refract Surg. 2007;33:418-429.

17. Alcon Laboratories. AcrySof IQ Toric Astigmatism IOL [package insert]. Fort Worth, TX: Alcon Laboratories; 2009.

18. Tetz MR, Werner L, Schwahn-Bendig S, Batlle JF. Prospective clinical study to quantify glistenings in new hydrophobic acrylic IOL. Presented at: ASCRS Symposium on Cataract, IOL and Refractive Surgery; April 3-8, 2009; San Francisco, CA.

19. Tognetto D, Toto L, Sanguinetti G, Ravalico G. Glistenings in foldable intraocular lenses. J Cataract Refract Surg. 2002;28:1211-1216.

20. Behndig A, Monestam E. Quantification of glistenings in intraocular lenses using Scheimpflug photography. J Cataract Refract Surg. 2009;35:14-17.
21. Colin J, Praud D, Touboul D, Schweitzer C. Incidence of glistening with the latest generation of yellow-tinted hydrophobic acrylic intraocular lenses. J Cataract Refract Surg. 2012;38:1140-1146.

22. Christiansen G, Durcan EJ, Olson RJ, Christiansen K. Glistenings in the AcrySof intraocular lens: pilot study. J Cataract Refract Surg. 2001;27:728-733.

23. Cisneros-Lanuza A, Hurtado-Sarrió M, Duch-Samper A, GallegoPinazo R, Menezo-Rozalén JL. Glistenings in the Artiflex phakic intraocular lens. J Cataract Refract Surg. 2007;33:1405-1408.
Clinical Ophthalmology

\section{Publish your work in this journal}

Clinical Ophthalmology is an international, peer-reviewed journal covering all subspecialties within ophthalmology. Key topics include: Optometry; Visual science; Pharmacology and drug therapy in eye diseases; Basic Sciences; Primary and Secondary eye care; Patient Safety and Quality of Care Improvements. This journal is indexed on

\section{Dovepress}

PubMed Central and CAS, and is the official journal of The Society of Clinical Ophthalmology (SCO). The manuscript management system is completely online and includes a very quick and fair peer-review system, which is all easy to use. Visit http://www.dovepress.com/ testimonials.php to read real quotes from published authors. 